\title{
Tendencias en los estudios brasileños sobre el cambio de normativa contable*
}

\author{
Trends in Brazilian Studies on Accounting Regulations Change \\ Tendências nos estudos brasileiros sobre a mudança de normativa contábil
}

\author{
Xiomara E. Vazquez Carrazana ${ }^{a}$ \\ Universidad Federal de Uberlândia, Brasil \\ xesther68@gmail.com \\ ORCID: http://orcid.org/0000-0002-2338-337X \\ Gilberto José Miranda \\ Universidad Federal de Uberlândia, Brasil \\ ORCID: http://orcid.org/0000-0002-1543-611X
}

DOI: https://doi.org/10.11144/Javeriana.cc21.tebc

\section{Resumen:}

Este artículo tiene como objetivo describir las tendencias en la producción científica brasileña en relación con la nueva normativa contable en el escenario económico nacional. Se emplearon elementos del método bibliométrico, mediante un proceso de recolección de datos en la plataforma electrónica Scientific Periodicals Electronic Library -SPELL-. Fueron seleccionadas revistas clasificadas como A2, B1 y B2 en el sistema Qualis (plataforma para la evaluación de los programas de post-graduación brasileños según su producción científica), en el periodo 2008-2017. El análisis realizado muestra el eminente carácter mercadológico del pensamiento de la época, en estrecha relación con la teoría de los mercados eficientes. Los artículos publicados marcan tendencias hacia el conservadurismo contable, la divulgación voluntaria y el análisis del desempeño económico.

Códigos JEL: G14, M20, M41

Palabras clave: estudios brasileños, normativa contable, convergencia contable.

\section{Abstract:}

This article aims to describe the trends in Brazilian scientific production regarding the new accounting regulations in the national economic scenario. It was used elements of the bibliometric method, through a process of collecting data in the Scientific Periodicals Electronic Library, SPELL. Papers classified as A2, B1, and B2 in the Qualis system (Brazilian platform for evaluating graduate programs based on their scientific production) were selected, for the period 2008-2017. The analysis shows an eminent market tendency in the thought of the time, in a closed relationship with efficient markets theory. Published articles set trends towards accounting conservatism, voluntary disclosure, and economic performance analysis.

JEL Codes: G14, M20, M41

Keywords: Brazilian studies, accounting convergence, Brazilian authors, accounting convergence.

\section{Resumo:}

Este artigo tem como objetivo descrever as tendências na produção científica brasileira em relação com a nova normativa contábil no cenário econômico nacional. Foram utilizados elementos do método bibliométrico, mediante um processo de coleta de dados na plataforma científica de periódicos eletrônicos Scientific Periodicals Electronic Library, SPELL. Foram selecionadas revistas classificadas como A2, B1 e B2 no sistema Qualis (plataforma para a avaliação dos programas de pós-graduação brasileiros segundo a sua produção científica), no período 2008-2017. A análise mostra o eminente caráter de mercado do pensamento da época, em estreita relação com a teoria dos mercados eficientes. Os artigos publicados marcam tendências orientadas ao conservadorismo contábil, à divulgação voluntária e à análise do desempenho econômico.

Códigos JEL: G14, M20, M41

Palavras-chave: s: estudos brasileiros, normativa contábil, autores brasileiros, convergência contábil.

Notas de autor

${ }^{a}$ Autora de correspondencia. Correo electrónico: xesther68@gmail.com 


\section{Introducción}

La contabilidad es un saber dinámico que se construye espacio-temporalmente, caracterizada por la evolución permanente de los elementos generales constitutivos de un saber disciplinal (Mejía, Mora, \& Montes, 2013). El sistema de información contable está influenciado por las condiciones macroeconómicas del país donde este es generado; en la medida que los países alcanzan diferentes estadios de desarrollo, exigen de cambios del proceso de contabilización (Schrickel, 1997; Ansótegui, 2010). Esta consideración no se hizo sentir hasta que la globalización diseminó la presencia de empresas extranjeras en diferentes países del mundo, lo que dio paso al proceso de convergencia a la International Financial Reporting Standard -IFRS-, con miras a la uniformidad del lenguaje contable (Rueda, 2010; Moreira \& Lima, 2003).

En Brasil, este proceso de convergencia estuvo marcado por varios acontecimientos: la creación del Comité de Pronunciamientos Contables -CPC-, por la Resolución 1.055 (2005) y la promulgación de la Ley 11.638 (2007); destacándose algunos antecedentes, tales como el Comunicado del Banco Central -BACEN14.259/06 (2006), la Instrucción de la Comisión de Valores Mobiliarios -CVM- 457/07 (2007) y la Circular de la Superintendencia de Seguros Privados -SUSEP- 357/07 (2007). En todos los casos, fueron tratados los aspectos específicos para divulgación de informaciones en el estándar IFRS (Alvaro et al., 2013).

Este contexto incentivó el desarrollo de estudios teórico-prácticos sobre la relevancia de la información contable para los usuarios externos, temática que ha dominado el escenario contable desde la migración hacia el valor justo como principal método de medición (Perea, 2015). El método de valor justo tiene un enfoque de mercado, convirtiéndose en una estrategia del proceso de internacionalización conducente a garantizar información común para los participantes de los mercados e imponiendo grandes retos de valuación y análisis (Arias \& Sánchez, 2011; Arias \& Sánchez, 2014). Así, el modelo tradicional de actividades contables, registro de partidas y eventos a su valor histórico de transacción, pasó a ser sustituido, siempre que fuera posible, por los valores del mercado; este cambio drástico en las normas contables no alcanzó el consenso pleno, y la controversia académica no se hizo esperar.

Los trabajos que defienden el "justo valor", argumentan que el criterio tradicional de costo histórico es un registro estático, principalmente para los activos no monetarios; contrariamente al "justo valor", que por traducir el valor de mercado considerado como "valor justo", ofrece una descripción más fidedigna del valor de la empresa (Pito \& Barros, 2016).

Una de las críticas más importantes es que los resultados financieros obtenidos mediante la contabilidad de valor justo contienen ganancias no realizadas, y al registrarlas se atenta contra uno de los principios más antiguos en contabilidad: el conservadurismo, que busca que la información contenida en los estados financieros no esté sobreestimada para no generar expectativas falsas que conduzcan a una inadecuada toma de decisiones de los usuarios de la información financiera (Perea, 2015).

A pesar de que el uso de este modelo contable encierra riesgos importantes (Silva, 2014; Pito \& Barros, 2016), su adopción en Brasil abrió mejores oportunidades para la inversión extranjera en el país (Zortea et al., 2017; Figlioli, Lemes \& Lima, 2017; Gonçalves, Conegliam \& Carmo, 2017), esto se vio reflejado en la literatura contable brasileña donde aumentaron las publicaciones que relacionaban indicadores contables y precio de las acciones; ayudando en la creación de campos de investigación que buscan contrastar el escenario anterior y pos-IFRS (Zortea et al., 2017).

Esto nos lleva a la pregunta de investigación que guía este estudio: ¿Cómo es abordado por los autores brasileños la repercusión del cambio de normativa contable?

A nivel internacional, la academia como primera organización en participar activamente en el estudio, discusión y construcción del modelo contable basado en el valor justo (Salazar, 2009), se pronunció caracterizando este modelo como un verdadero desafío con importantes cambios para la doctrina contable (Cano, 2010), destacando la importancia de estudios sobre estos temas en la diversificación del proceso de 
investigaciones en esta área (Torres \& Rodríguez, 2008; Macías \& Cortés, 2009; Ruiz, 2008; Sánchez \& Salazar, 2010; Sosa, 2013).

Las revistas líderes de contabilidad, positivistas y cuantitativas, se centraron en resultados empíricos, evidenciando un posicionamiento epistemológico y metodológico particular de la comunidad científica en la evaluación de cambios de la calidad de la información contable (Gómez, 2013). Un análisis sobre los artículos publicados en revistas pertenecientes al Qualis de Brasil permitirá explorar el pensamiento brasileño en torno al impacto que produce el cambio de normativa contable.

El objetivo de la presente investigación consiste en revelar las tendencias marcadas por los autores brasileños en los estudios sobre la repercusión del cambio de normativa contable.

Ante el profundo dinamismo de la disciplina contable en un entorno económico-social cambiante se justifica la realización de un estudio sobre tendencias del pensamiento contable. Una recopilación de ideas en el horizonte temporal definido se convierte en un instrumento fundamental para controlar el avance del conocimiento científico de esta rama del saber, lo que abrirá espacios a enjuiciamientos críticos y al desarrollo de instrumentos empíricos pertinentes para la transformación hacia resultados esperados y necesarios.

\section{Metodología}

Este estudio utiliza elementos del método bibliométrico para analizar la diseminación del conocimiento científico sobre la repercusión del cambio de normativa contable en Brasil. Fue importante mapear las principales motivaciones de la comunidad académica de contabilidad del país, a través de los estudios publicados, para inducir los principales temas de investigación que pueden asociarse al proceso de convergencia y armonización de la normativa contable que comienza en 2008 con un período de tránsito hasta el 2009; a partir del año 2010 las compañías de capital abierto deben de presentar sus informaciones financieras de acuerdo con la nueva normativa contable. Esto justifica el período de recolección de los datos de estudio, enmarcado en 2008-2017.

Se realizó un proceso de recolección de datos en la plataforma electrónica Scientific Periodicals Electronic Library -SPELL-, a través de la búsqueda avanzada en los campos “Título del documento", "Resumen", "Palabras claves", utilizando términos relacionados con el asunto propuesto: convergencia de las normas internacionales, IAS; estandarización, IFRS; estándar contable; estándar internacional; adopción IFRS, CPC; armonización, 11.638; IASB; armonización 11.941; IPSAS (International Public Sector Accounting Standard); IAESB (International Accounting Education Standard Board) y GLENIF (Grupo Latinoamericano de Emisores de Normas Financieras Internacionales). Vale mencionar que la búsqueda de estos términos se realizó de manera individual.

Se revisaron, inicialmente, 513 artículos científicos publicados en revistas de la base de datos brasileña SPELL. Una vez filtrados los artículos teniendo en cuenta los años de mayor interés y su relación directa con el tema de impacto de las normas sobre los diferentes eventos contables, la muestra de artículos a ser evaluados pasó a ser de 337 artículos.

Las revistas empleadas fueron las clasificadas en el Qualis de Brasil aceptado para la evaluación de los programas de post-graduación: Qualis A1; Qualis A2; Qualis B1 y Qualis B2. El primer Qualis no está representado en el estudio porque no hay revistas brasileñas del área contable en esta clasificación. El 68\% de los artículos fueron publicados en las revistas de mayor ranking ( $\mathrm{A} 2$ y $\mathrm{B} 1$ ), lo cual muestra la relevancia de las temáticas abordadas.

Para el análisis de los datos y la presentación de los resultados, los artículos se organizaron por grupos temáticos, que se construyeron atendiendo a la lógica con la que fue abordada la temática en las producciones científicas revisadas. Se detectó la existencia de un período valorativo y crítico sobre la nueva normativa y 
aquí se concentraron diversos estudios enfocados en el análisis de la internacionalización y su impacto en la información contable.

Estos estudios trazaron diversas rutas que permitieron explorar el tema desde diversas perspectivas, señalando un terreno de análisis de problemas orientados a la evaluación del impacto producido en grupos de cuentas específicos. Frente a las posturas críticas del primer grupo de artículos, un grupo de autores despliegan una mirada analítica que intenta buscar explicaciones al vínculo de diferentes prácticas contables tradicionales ante la nueva postura: mercados eficientes.

\section{Generalidades sobre el pensamiento de académicos contables en Brasil}

La revisión de estudios en contabilidad muestra una baja diversidad temática, metodológica y epistemológica (Mendonça, Riccio \& Sakata, 2009; Nascimento, Junqueira \& Martins, 2010; Homero, 2017). La ausencia de una línea consolidada, interpretativa y crítica (Lima, 2011; Rosa \& Alcadipani, 2013) condiciona un proceso de investigación estéril y estancado (Major, 2017).

En la producción científica brasileña de finales de la década de 1990 prevaleció un discurso erudito, en lugar de un campo científico (Homero, 2017). Para Bourdieu (1976), un campo científico es una red de configuración de relaciones objetivas entre posiciones con disputa por el monopolio de la autoridad científica o capacidad legítima de expresión en materia de ciencia; de manera que un campo científico será aquel que por su alto grado de autonomía puede adoptar estrategias de conservación de determinadas ideas científicas.

En la contabilidad brasileña, Homero (2017) entiende que el campo científico tiene una baja autonomía en relación con el campo profesional, razón por la cual prevalece un carácter mercadológico y monoparadigmático en el quehacer científico de académicos e investigadores de esta disciplina.

Los estudios realizados en la década del 2000 se enfocan hacia el análisis de la información contable en el contexto de mercados eficientes; esta escasa diversidad es consecuencia de la baja autonomía del campo científico condicionado por el abordaje positivista de las revistas académicas más destacados, que provoca la reproducción del paradigma que prevalece y, con ello, la configuración del campo científico que monopoliza la práctica contable (Zortea et al., 2017; Homero, 2017).

Las decisiones estratégicas de las empresas relacionadas con la asociación, fusión u otro tipo de transformación en sociedades marcaron el rumbo de la economía hacia la internacionalización del capital y el auge de la economía de mercado; en tal sentido, los autores se pronuncian por una homogenización de procedimientos contables para garantizar la eficiente utilización de mecanismos financieros como base del desarrollo de los mercados de capitales (Campos \& Scherer, 2001; Niyama et al., 2002; Moreira \& Lima, 2003).

La sofisticación e integración de estos mercados fue un factor decisivo para el auge de discusiones sobre las divergencias de las prácticas contables y su impacto sobre la transparencia y precisión de información para los accionistas (Silva et al., 2003). El segmento académico estrechó vínculos con los temas basados en el mercado de capitales, y la academia brasileña se inclinó hacia el campo conocido como value relevance, problema de investigación limitado a acreedores e inversores como público de interés (Homero, 2017).

La práctica investigativa con un marcado enfoque hacia el mercado se convirtió en tema central de la teoría y la práctica contable brasileña desde la última parte del siglo XIX (Alvaro et al., 2013), manifestación de que no solo las prácticas sociales son el reflejo de los modos de actuación de los individuos, sino que los individuos acaban adaptándose e incorporando a sus prácticas las exigencias y posturas económico-sociales de una época. En la lucha por la legitimidad científica, la tendencia es a adoptar estrategias conservadoras perpetuando el orden científico establecido por los medios -en este caso, las revistas- encargados de la producción y circulación de ideas científicas (Homero, 2017). 
La eficiencia del mercado de capitales constituye la teoría motivadora para evaluar la dinámica inherente al comportamiento de los indicadores del desempeño empresarial como previsores del precio de las acciones. Este tipo de investigación procura identificar los factores del desempeño relevantes para anticipar los movimientos del precio de las acciones. De esta forma, los estudios direccionados a corroborar o refutar presencia de causalidad entre indicadores de mercado y desempeño económico ofrecen evidencias relevantes y originales de integración de estos dos espacios (Carvalho, Costa \& Goulart, 2008; Callado et al., 2010; Cerqueira et al., 2012; Fé Júnior, Nakao \& Ribeiro, 2015; Rezende, Almeida \& Lemes, 2015).

El enfoque de mercado contribuyó para que otros temas comenzaran a recibir atención dada su relación con la confiabilidad y la neutralidad de la información contable: el conservadurismo y la divulgación voluntaria.

El conservadurismo contable fue uno de los atributos utilizados para medir la calidad de la información contable; de acuerdo con los principales hallazgos la información contable resulta ser de mayor calidad porque anticipa posibles salidas de flujos de efectivos futuros preservando el efectivo de los stakeholders (Santos et al., 2011; Silva, 2011; Scalzer, Beiruth \& Reina, 2017; Santiago, Cavalcante \& Paulo, 2015; Vale \& Nakao, 2017; Lourenço \& Branco, 2015; Souza \& Lemes, 2016; Oliveira, Nakao \& Nardi, 2017; Sousa et al., 2018).

Muchos autores resaltan que la presencia de conservadurismo es un denotativo de calidad de la utilidad (Francis et al., 2005) y reduce la posibilidad de manipulación de los resultados para maximizar el desempeño económico, por esta razón constituye una herramienta reductora de asimetría informacional; siendo la asimetría informacional proporcional al conservadurismo (Lafond \& Watts, 2008).

Algunos estudios analizaron la utilidad y relevancia del conservadurismo como práctica, sus resultados sugieren que la utilidad más conservadora tiene mayor grado de relevancia en el valor de la empresa; el conservadurismo impactó el comportamiento de los analistas de mercado después de adoptadas las IFRS, mejorando sus previsiones e intereses; esto contribuyó a la reducción de la volatilidad del retorno de las acciones. Así, diferentes autores infirieron que el conservadurismo contable mejora el ambiente de la información empresarial (Lara, Osma, \& Penalva, 2014); mientras que, en el estudio de Silva, Heinzen, Klan y Lemes (2018), se observó que el conservadurismo contable se asoció negativamente con la valorización de las empresas en el mercado; lo que evidenció -en este estudio- la falta de relevancia de esta práctica para los usuarios de la información. Algunas investigaciones dejan abiertas discusiones sobre si el mercado ofrece más importancia para informaciones más o menos conservadoras.

La divulgación de información voluntaria pretende ofrecer mayor transparencia a los inversores y promover más transparencia y reducción de asimetría informacional (Schwarz, Barbosa \& Freitas, 2019). Aunque es sabido que la divulgación voluntaria trae beneficios (mayor liquidez de mercado de los títulos negociables y menor costo de capital); la problemática en torno a este tema radica en que los gestores no siempre están dispuestos a aumentar el nivel de divulgación de las informaciones ya que pueden existir elementos más competitivos que justifiquen un control gerencial más rígido sobre las informaciones, contribuyendo a la importancia de la oportunidad de divulgarlas o no (Consoni, Colauto \& Lima, 2017).

Algunos estudios demostraron que la divulgación voluntaria contribuye a la reducción o eliminación de la asimetría informacional, y una baja asimetría informacional dificulta el gerenciamiento de resultados. Este tipo de investigaciones enfrentan dificultades para obtener una medida apropiada de la divulgación; los investigadores escogen sus propias medidas por lo que este proceso no está desprovisto de subjetividad. Se deben discutir varias cuestiones: la literatura actual es dispersa y con diferencias en el entendimiento conceptual de que la divulgación voluntaria es una respuesta a la asimetría informacional (Consoni et al., 2017). 


\section{Temáticas abordadas y principales tendencias}

En la tabla 1 se presenta la distribución de las principales temáticas abordadas, por artículos y por los diferentes años que componen el período en estudio.

TABLA 1

Agrupamiento de artículos por temas de investigación

\begin{tabular}{|l|c|c|c|c|c|c|c|c|c|c|c|}
\hline \multicolumn{1}{|c|}{ Temáticas } & 2008 & 2009 & 2010 & 2011 & 2012 & 2013 & 2014 & 2015 & 2016 & 2017 & Total \\
\hline $\begin{array}{l}\text { 1. Generalidades sobre el proceso de } \\
\text { cambio }\end{array}$ & 10 & 9 & 6 & 2 & 8 & 14 & 15 & 17 & 13 & 13 & 107 \\
\hline $\begin{array}{l}\text { 2. Impacto en procesos contables } \\
\text { especificos }\end{array}$ & 3 & 6 & 3 & 5 & 12 & 16 & 19 & 15 & 5 & 2 & 86 \\
\hline 3. Proceso de enseñanza-aprendizaje & 1 & 2 & 3 & 2 & 1 & 3 & 2 & 2 & 1 & 2 & 19 \\
\hline 4. Conservadurismo contable & 1 & - & 1 & 2 & - & 1 & 2 & 3 & 6 & 3 & 19 \\
\hline 5. Divulgación voluntaria & - & 2 & 2 & 4 & 3 & 4 & 3 & 1 & 4 & 2 & 25 \\
\hline $\begin{array}{l}\text { 6. Impacto sobre el desempeño } \\
\text { económico }\end{array}$ & - & 6 & 5 & 2 & 9 & 9 & 17 & 15 & 8 & 10 & 81 \\
\hline \multicolumn{1}{|c|}{ Total } & 15 & 25 & 20 & 17 & 33 & 47 & 58 & 53 & 37 & 32 & 337 \\
\hline
\end{tabular}

Fuente: elaborada por los autores.

La información que muestra la tabla 1 permite afirmar que el período comprendido entre 2011-2014 marca una tendencia creciente en la publicación de artículos sobre el impacto de las IFRS, con una disminución de artículos publicados durante 2016 y 2017. El auge de publicaciones de período 2011-2014, responde a la necesidad de la valoración de las consecuencias prácticas de la adopción de las IFRS y con ello la caracterización del impacto de esta nueva normativa; más del $50 \%$ del total de publicaciones fueron dedicadas a valoraciones generales y específicas del impacto en algunas partidas y eventos contables. Por esto se puede inferir que ocurrió un estancamiento en la construcción de nuevo conocimiento.

La mayoría de los artículos publicados de los años 2008 y 2009 se concentraron en los temas más generales; muchas investigaciones atendieron el tema de la evolución del concepto del valor justo en las normas brasileña con un análisis gradual e histórico del desarrollo de estas normas dentro del concepto fair value (Castro, Morch, \& Marques, 2008; Murcia et al., 2008), algunos autores escribieron sobre las inferencias económicas, a priori, de las reglamentaciones nacionales en consonancia con los estándares internacionales (Fernandes et al., 2008; Ito, Mendes \& Niyama, 2008; Strouhal, 2009; Alves \& Moreira 2009; Pereira \& Marques, 2009; Barbosa, Dias \& Pinheiro, 2009; Silva, Couto \& Cordeiro, 2009; Fuji, 2008; Santos \& Cia, 2009).

En el período 2009-2013 se destacan los estudios que afirman que la convergencia para los estándares contables internacionales es un asunto relevante, desarrollando comparaciones de los pronunciamientos técnico CPC con cada una de las Normas Internacionales, con el objetivo de verificar diferencias entre estos dos sistemas de reglas. Esto dio lugar a algunas discusiones sobre las ventajas y desventajas de una normativa contable basadas en reglas o en principios (Dias, Cunha \& Mário, 2009; Dantas et al., 2010; Costa, Theóphilo \& Yamamoto, 2012).

Obsérvese en la tabla 1, que estos temas generales merecen la mayor atención a partir del año 2013, tres años después de tornarse obligatorias las nuevas normas. Los estudios comienzan a argumentar que esa nueva normativa contable mejora la calidad de la información contable y las torna más comparables y transparentes. Estas aseveraciones fueron posibles mediante la verificación de los cambios en el nivel de gerenciamiento de resultados después de la adopción de la normativa (Grecco, 2013; Domingos, Lima \& Ponte, 2013; Joia \& Nakao 2014; Barreto, Santos \& Tavares, 2017).

Algunos estudios se desarrollaron con el objetivo de analizar el impacto de los factores: 1) método contable en uso; 2) características culturales, económicas y sociales; 3 ) incentivos de las empresas sobre la efectividad del proceso de adopción de las normas internacionales (Santos \& Silva, 2014; Machado \& Nakao, 2014; Tavares, Anjos \& Paulo, 2014). 
En esta etapa mereció la atención, además, temas relacionados con los obstáculos enfrentados por diferentes grupos de stakeholders para lograr una plena armonización contable a partir de la participación de estos, en el proceso de emisión/alteración de estas normativas (Carvalho et al., 2015; Haveroth et al., 2017), y otros estudios sobre la relación de factores subjetivos y de contingencias en la aplicación del valor justo (Klein \& Almeida, 2017; Silva Filho, Lucena \& Leite Filho, 2017).

De forma simultánea, y con una mayor representatividad de los artículos en la etapa 2012-2015, se desarrollaron investigaciones valorativas sobre procesos contables específicos (relacionados con la aplicación concreta de los pronunciamientos técnicos- CPC). Cabe destacar que en los años 2013 y 2014 los artículos relacionados con estas temáticas acapararon la atención de los investigadores y académicos.

Los estudios sobre activos intangibles (CPC-04) analizaron el impacto de la nueva normativa sobre la presentación de este rubro, se confirmó que los sectores que invierten en mejores tecnologías y con una concentración de capital más alta son más propensos a divulgar información voluntaria sobre los activos intangibles (Camargos, Taboada \& Moura, 2012; De Menesse, Rodrigues \& Vasconcelos, 2013; Klann et al., 2014; Mantovani \& Almeida, 2014; Dias, Siqueira, Beuren, 2014; Margarete \& Klann, 2015).

Un hecho que suscitó la ocurrencia de diversos estudios prácticos fue la puesta en práctica del International Accounting Standards 41-Agriculture (IAS 41) y en consecuencia, en Brasil, el CPC-29 (Registro de Activo Biológico y Producto Agrícola). El objetivo fundamental de esa reglamentación es reducir las diferencias en la forma de contabilización de los eventos relacionados con la producción y transformación de activos biológicos; este tipo de aplicación ha sido un tema ampliamente tratado.

La novedad de que los activos biológicos sean medidos por su valor justo en la fase de transformación hasta la colecta y que los resultados obtenidos en este proceso de transformación deberán ser evidenciados en el período cuando ocurren y no de acuerdo con el período del ciclo operacional, como normalmente era utilizado y permitido, es un tema que ha provocado amplios debates. Las discusiones versan alrededor del potencial informativo y las limitaciones de uso de regulaciones referidas a este particular, cuando la adopción del valor justo para la medición de plantas y animales ocurre en un contexto de mercado activo inexistente (Rech et al., 2008; Einsweiller \& Fischer, 2013; Da Cunha, Gomes, \& Veras, 2013; Dalmutt et al., 2014; Souza \& De Lima, 2014; Talaska, \& De Lima, 2016; Ramos \& Da Silva, 2017).

El impacto de la nueva normativa para el registro de los Activos Inmovilizados (CPC 27) y Propiedades de Inversión (CPC 28), también fueron objeto de diferentes estudidos empíricos (Fé, Vaz de Lima \& Oliveira, 2012; Costa, Silva \& Laurencel, 2013; Moraes \& Carvalho, 2014; Poli, Doná \& Neumann, 2014; Silva, Fonseca \& Nogueira, 2014; Peruchena, Shneider \& Kronbauer, 2015).

El despliegue de esta temática en el país fue notorio y el análisis de la vinculación del proceso de enseñanzaaprendizaje con estos temas no se hizo esperar. A lo largo de este momento histórico se pusieron de relieve algunos temas de análisis de los CPC y su relación con las Normas Internacionales de Contabilidad para los cursos de graduación en el país (Sá \& Malaquias, 2012).

Diferentes propuestas para el perfeccionamiento de los programas y currículos de los programas de postgraduación, surgieron a partir de la identificación de la percepción de los profesores sobre el abordaje de estos temas en un escenario de convergencia a las Normas Internacionales de Contabilidad (Cunha, Guidini, \& Klann, 2017; Alves et al., 2017).

A pesar de la riqueza de los análisis realizados en las temáticas anteriores (las tres primeras temáticas de la tabla 1), el enfoque empleado no permitió concentrarlas dentro de la línea de pensamiento más delineada por los autores de la época. Las tendencias más evidentes surgen a partir del tratamiento científico de la dualidad entre: argumentos que apuntan hacia un aumento de la calidad de la información contable y el peligro de eliminar algunas alternativas contables que reflejen de forma más adecuada la posición patrimonial de las empresas (Lourenço \& Branco, 2015; Souza \& Lemes, 2016).

Relacionado con esto, algunos autores sintieron preocupación acerca del conservadurismo contable (Santos et al., 2011; Silva, 2011; Scalzer et al., 2017; Santiago et al., 2015; Vale \& Nakao, 2017). El objetivo 
de las normas actuales está orientado, esencialmente, para el futuro; una consecuencia importante es que la subvaloración en períodos actuales puede conducir a una sobrevalorización en períodos futuros (Scalzer et al., 2017). Esto puede estar indicando una mirada diferente sobre las métricas para la evaluación de la calidad y el conservadurismo (Vale \& Nakao, 2017).

Otro aspecto importante a ser considerado es la escasa orientación detallada para la implementación de las IFRS, lo que permite mayor poder de arbitrariedad al gestor y favorece la aparición o consolidación de elementos que pueden perjudicar la homogenización en el reflejo de la posición patrimonial, y con ello importantes afectaciones a la eficiencia del mercado de capitales (Lourenço \& Branco, 2015; Souza \& Lemes, 2016; Oliveira et al., 2017).

La información contable y el mercado de capitales son asuntos íntimamente relacionado; la primera expresión contempla el conjunto de informaciones utilizadas por los agentes que actúan en el mercado para subsidiar el proceso decisorio. Sin embargo, algunas divergencias de intereses y el incentivo de la arbitrariedad, son manifestación de asimetría informacional (Magalhães, Pinheiro \& Lamounier, 2011).

Dado que este elemento provoca pérdida de confianza de los accionistas y, consecuentemente, un éxodo de estos hacia otros mercados más seguros, a fin de proteger su capital; esta temática despertó el interés de la comunidad académica (Araujo \& Reis, 2013), considerándose este aspecto como uno de los grandes desafíos de la normatización contable (Klann \& Beuren, 2010) y la primicia para que los estudios sobre divulgación voluntaria alcanzaran notable visibilidad en las publicaciones brasileñas (Magalhães et al., 2011; Loureiro, Gallon \& Luca, 2011; Maia, Formigoni \& Silva, 2012; Silva et al., 2017).

La consideración de que la adopción de pobres prácticas de transparencia y revelación de información, podría generar graves problemas de asimetrías de información y con ello, fuertes afectaciones a la eficiencia de los mercados; fue una arista que desbordó los problemas abordados en la época (Silva \& Nakao, 2011).

En ese contexto, surgieron los estudios sobre Divulgación Voluntaria. El mercado de capitales brasilero tiene ciertas especificidades que lo distinguen de otros mercados, presenta un débil enforcement, es regido por el modelo stakeholder, manifiesta alta volatilidad, influencia gubernamental y concentración accionaria con presencia de estructuras piramidales (Silveira et al., 2009; Lopes \& Alencar, 2010), es por esta razón que existen conjeturas de que el proceso de convergencias de normas internacionales sometió a las empresas brasileñas a un nuevo régimen de exigencias de divulgación obligatoria que puede haber alterado significativamente el nivel de divulgación voluntaria (Consoni \& Colauto, 2016).

La divulgación voluntaria procura aumentar la visibilidad y la valorización de la empresa hacia el mercado, es un comportamiento estratégico de las empresas direccionado hacia la percepción de su valor por parte del mercado. Las empresas pueden aumentar su divulgación voluntaria con el fin de disminuir su asimetría informacional, aumentar transparencia y disfrutar de los beneficios de mayor liquidez de las acciones y menor costo de capital (Lopes \& Alencar, 2010; Consoni \& Colauto, 2016).

La literatura especializada dio señales de existencia de una cierta confusión contable en cuanto a las alteraciones expresivas del patrimonio líquido y la utilidad en empresas de Brasil como consecuencia de los cambios de normativa (Santos, 2015). Se abrió un espacio importante a las discusiones sobre el impacto de la normativa sobre el desempeño empresarial, y aparecieron estudios empíricos sobre el cambio del patrimonio, las ventas y la utilidad después de la adopción las IFRS (Da Cunha et al., 2013; Daza, 2015; Pires et al., 2017).

La postura de claro liderazgo asumida por los estudios basados en el impacto de la normativa sobre el desempeño económico a partir de 2010, estuvo condicionada por el propio objetivo de la adopción de la normativa contable, centrado en que la contabilidad ofrezca un signo positivo en dirección a una mayor calidad y transparencia (Zonatto et al., 2011; Almeida et al., 2011, Santos, 2012; Silva, Macedo \& Marques, 2012; Costa, Almeida \& Silva, 2013; Macedo, Machado \& Machado, 2013; Pires \& Decourt, 2015; Cardoso, Souza \& Dantas, 2015 ; Moura \& Coelho,2016; Sayed et al., 2013; Silva et al., 2017; Nunes, Funchal \& Beiruth, 2017). 
El uso de la utilidad ha sido uno de los indicadores más empleados para demostrar el impacto de las normas sobre el resultado del desempeño empresarial (Estima \& Mota, 2015; Luzivalda \& Edilson, 2017). Por otro lado, algunos autores demuestran empíricamente que las IFRS impactan la calidad de los resultados de la empresa, tornándose la información contable más persistente y relevante (Brunozi et al., 2017), mientras que otros estudios indican que no se percibe mejoría en la calidad en la información contable dada la dispersión y pocas posibilidades de previsión (De Aguiar \& Hiroshi, 2017). El desempeño económico en su integralidad es una de las cuestiones más controvertida en este tipo de estudio (Pires et al., 2017).

Las investigaciones realizadas sobre este tema tienen como objetivo principal regular la información contable y con esto mitigar la asimetría de información entre los diferentes mercados. Los argumentos sugieren que la adopción de las IFRS produce beneficios significativos para el mercado de capitales, partiendo de la premisa de que la divulgación de esta nuevas normativas aumentan la transparencia y la calidad de los Estados Financieros porque estos estándares están más orientados al mercado de capitales (Black \& Nakao, 2017; Zortea et al., 2017).

\section{Reflexiones}

La adopción de la nueva normativa contable abrió un campo para las investigaciones brasileñas: el tema de asimetría informacional fue el punto de partida para el despliegue de las investigaciones contables de los académicos de esta rama que rápidamente develaron su interés científico hacia el campo conocido como value relevance.

La línea general abordada dentro de los estudios brasileños, relacionados con el tema de las IFRS, estuvo direccionada a la constatación de que la adopción obligatoria de una nueva normativa contable pasó a estar significativamente asociada con una mayor eficiencia de capital invertido por los accionistas, abordaje que fue considerado importante en su contribución al desarrollo de los mercados de capitales como impulsores del desarrollo de la economía de Brasil y la región.

Una de las principales aseveraciones de los investigadores sobre el tema consiste en que la eficiencia de la información en el mercado de capitales se traduce en la velocidad con que las informaciones disponibles a los usuarios repercuten en los precios de las acciones y en las expectativas de los accionistas, promoviendo la idea de que la reformulación de los informes financieros impacta el mercado de capitales.

Sin embargo, el carácter mercadológico de base en las investigaciones de la época reveló posturas y líneas de pensamientos diferentes. Las principales preocupaciones abordadas por los autores, la intensidad de los efectos directos sobre el objeto de estudio, la problematización y claras evidencias de brechas teóricometodológicas para la continuidad, permitieron la definición de algunas tendencias.

Algunos autores concentraron esfuerzos en el análisis de divulgación voluntaria y conservadurismo, evidenciándose afectaciones a la divulgación voluntaria después del proceso de adopción de las IFRS. Esto abrió espacio para los estudios sobre tratamientos más específicos a Disclosure compulsorio a partir de normativas específicas.

El conservadurismo es uno de los temas que se ha mostrado más inconcluso. Los estudios han sido heterogéneos, no existen suficientes evidencias que confirmen aumento de conservadurismo contable después de la adopción de las IFRS; algunos resultados empíricos denunciaron insuficiencias en la manifestación de este atributo, mientras que otros constatan presencia de reconocimiento más tempestivo de las pérdidas que de las utilidades después de adoptadas las nuevas normativas.

Otro grupo importante de autores delimitó su propósito a entender y evidenciar cómo los informes financieros reflejan el valor de la empresa, lo cual intensificó la relevancia de los indicadores del desempeño económico en un nuevo escenario contable. Esto develó una corriente principal de análisis sustentada en la capacidad asociativa entre indicadores de desempeño y valor de mercado, con énfasis en los análisis de la 
tempestividad de la información contable o capacidad de capturar y expresar, contemporáneamente, eventos considerados relevantes por el mercado.

Fueron diversos y significativos los artículos que evidenciaron diferencias de la relevancia informacional de indicadores económicos en los períodos pre y pos-primera fase del proceso de convergencia a las normas internacionales. Esto permite conjeturar sobre el surgimiento de nuevas ideas epistemológicas y metodológicas que desplegarán diferentes interrogantes para propiciar la incorporación gradual de nuevos métodos de análisis. Se señala un camino a seguir: la articulación de nuevos fundamentos teóricos que permitan la delimitación de otros enfoques y justifique una base crítica para determinar otras conductas y núcleos para creación de grupos temáticos que contribuyan al direccionamiento de las ideas científicas.

\section{Referencias}

Almeida, S., Silva, A., Costa, T. \& Laurencel, L. (2011). Análise dos impactos das normas internacionais de contabilidade sobre o lucro líquido e o patrimônio líquido das empresas do setor de extração e processamento de recursos naturais. Revista de Contabilidade do Mestrado em Ciências Contábeis da UERJ, 16(3), 136-156. http: //www.spell.org.br/documentos/ver/8825

Alvaro, M., Reis, M., Veras, M. \& Cardoso, P. (2013). Impacto de la convergencia a las Normas Contables Internacionales en el Brasil sobre el contenido informacional de la contabilidad. Revista de Educação e Pesquisa em Contabilidade, 7(3), 223-240. http://www.repec.org.br/index.php/repec/article/download/905/764

Alves, D., Kronbauer, C., Ott, E. \& Thomaz, J. (2017). O Ensino dos CPCs nos cursos de Ciências Contábeis em Instituições de Ensino Superior do Brasil. Revista Contemporânea de Contabilidade, 14(32), 48-70. http://dx.d oi.org/10.5007/2175-8069.2017v14n32p48

Alves, P. \& Moreira, J. (2009). The adoption of the international financial reporting standards in Portugal: Can expected costs be reduced? Revista Universo Contábil, 5(3), 156-164. http://dx.doi.org/10.4270/ruc.20095

Ansótegui, A. (2010). Convergencia internacional en materia de subvenciones: referencia a los Derechos de Emisión de Gei. Revista Universo Contábil, 6(1), 156-170. http://www.spell.org.br/documentos/ver/6243

Araujo, L. \& Reis, M. (2013). A produção científica sobre assimetria informacional em periódicos internacionais de contabilidade. Revista de Contabilidade e Controladoria, 5(1), 99-119. http://dx.doi.org/10.5380/rcc.v5i1.299 23

Arias, M. \& Sánchez, A. (2011). Valuación de activos: una mirada desde las Normas Internacionales de Información Financiera, los Estándares Internacionales de Valuación y el contexto actual colombiano. Cuadernos de Contabilidad, 12(30), 95-126. http://revistas.javeriana.edu.co/index.php/cuacont/article/view/3112

Arias, M. \& Sánchez, A. (2014). Bases de medición: correspondencia entre las Normas Internacionales de Información Financiera, los Estándares Internacionales de Valuación y el contexto actual colombiano. Cuaderno de Contabilidad, 15(37), 25-53. http://revistas.javeriana.edu.co/index.php/cuacont/article/view/9001

Barbosa, J., Dias, W., \& Pinheiro, L. (2009). Impacto da convergência para as IFRS na análise financeira: um estudo em empresas brasileiras de capital aberto. Contabilidade Vista \& Revista, 20(4), 131-153. http://www.spell.org .br/documentos/ver/8130

Barreto, D., Santos, R. \& Tavares, M. (2017). Uma Análise de Conteúdo das Cartas Comentários Enviadas por Empresas para as Alterações da Seção 1 - Objetivo da elaboração e divulgação de relatório contábil-financeiro de propósito geral da estrutura conceitual proposta pelo IASB. Revista de Gestão, Finanças e Contabilidade, 7(2), 176-196. http://dx.doi.org/10.18028/2238-5320

Black, R. \& Nakao, S. (2017). Heterogeneidade na qualidade do lucro contábil entre diferentes classes de empresas com a adoção de IFRS: evidências do Brasil. Revista Contabilidade \& Finanças - USP, 28(73), 113-131. http://dx.d oi.org/10.1590/1808-057x201702750

Bourdieu, P. (1976). Le champ scientifique. Actes de la Recherche en Sciences Sociales, 2, 88-104. https://doi.org/10.3 406/arss.1976.3454 
Brunozi, A., Coelho, D., Ott, E., Arenhal, L. \& Lima, F. (2017). Persistência e value relevance dos resultados contábeis com a adoção das IFRS: um estudo em empresas de países do GLENIF. Ref. Cont. 36(3), 33-51. http://dx.doi .org/10.4025/enfoque.v36i3.31820

Callado, A., Moller, H., Callado, A., \& Leitão, C. (2010). Relações entre índices de mercados acionários: uma análise empírica a partir da ótica da causalidade. Reuna, 15(3), 54-68. http://www.spell.org.br/documentos/ver/5566

Camargos, B., Taboada, L. \& Moura, W. (2012). Evidenciação de Ativos Intangíveis: Estudo Empírico em Companhias Abertas, Revista de Contabilidade e Organizaçôes, 6(14), 22-45. http://www.spell.org.br/documentos/ver/7628

Campos, G. \& Scherer, L. (2001). Lucro por ação. Revista Contabilidade \& Finanças - USP, 12(26), 81-94. http://w ww.spell.org.br/documentos/ver/24085

Cano, A. (2010). Análisis de la norma internacional de contabilidad (NIIF/NIC) No. 8 (Políticas contables, cambios en las estimaciones contables y errores) y sus principales efectos en el Estatuto Tributario colombiano. Cuadernos de Contabilidad, 11(28), 123-147. Disponible en: http://revistas.javeriana.edu.co/index.php/cuacont/article/ view/3200

Cardoso, R., Souza, F. \& Dantas, M. (2015). Impactos da Adoção do IFRS na Acumulação Discricionária e na Pesquisa em Gerenciamento de Resultados no Brasil. Revista Universo Contábil, 11(2), 65-84. http://dx.doi.org/10.427 $0 /$ ruc.201521

Carvalho, C., Albuquerque, F., Quirós, J. \& Justino, M. (2015). Uma Análise das Diferenças em Termos dos Interesses Profissionais a Partir do Projeto de Substituição da IAS 39. Revista Contabilidade \& Finanças - USP, 26(68), 181-194. http://dx.doi.org/10.1590/1808-057x201500530

Carvalho, P., Costa, A., \& Goulart, A. (2008). Analise técnica versus hipótese dos mercados eficientes: um estudo utilizando o indicador MACD. Revista Alcance, 15(3), 397-415. http://www.spell.org.br/documentos/ver/267 3/analise-tecnica-versus-hipotese-dos-mercados-eficientes--um-estudo-utilizando-o-indicador-macd/i/pt-br

Castro, V., Morch, R., \& Marques, J. (2008). Fair value Accounting: uma análise sobre a evolução do conceito de valor justo entre as normas internacionais e as normas brasileiras. Sociedade, Contabilidade e Gestão, 3(1), 112-126. h ttp://www.spell.org.br/documentos/ver/34214

Cerqueira, D., Rezende, A., Dalmácio, F., \& Silva, J. (2012). O impacto do reconhecimento do custo atribuído e da divulgação de impairment de ativos tangíveis e intangíveis (IFRS) sobre os preços e os retornos das ações das companhias brasileiras. Revista de Contabilidade do Mestrado em Ciências Contábeis da UERJ, 17(3), 5-23. htt p://www.spell.org.br/documentos/ver/9331

Consoni, S. \& Colauto, R. (2016). A divulgação voluntária no contexto da convergência às Normas Internacionais de Contabilidade no Brasil. Revista Brasileira de Gestão de Negócios, 18(62), 658-677. http://dx.doi.org/10.7819 /rbgn.v18i62.2242

Consoni, S., Colauto, D., \& Lima, F. (2017). A divulgação voluntária e o gerenciamento de resultados contábeis: evidências no mercado de capitais brasileiro. Revista Contabilidade \& Finanças - USP, 28(74), 249-263. https: //doi.org/10.1590/1808-057x201703360

Costa, J., Theóphilo, C., \& Yamamoto, M. (2012). A aderência dos Pronunciamentos Contábeis do CPC às normas internacionais de contabilidade. Contabilidade, Gestão e Governança, 15(2), 110-126. https://cgg-amg.unb.br/ index.php/contabil/article/view/482

Costa, T., Almeida, S., \& Silva, A. (2013). Uma análise dos impactos da adoção das Normas Internacionais de Contabilidade no Brasil: um estudo de empresas de capital aberto no setor de bebidas, alimentos e comércio. Pensar Contábil, 15(56), 4-13. http://www.atena.org.br/revista/pensarcontabil/article/view/1539/1376

Costa, T., Silva, A. \& Laurencel, L. (2013). Escolha de práticas contábeis: um estudo sobre propriedades para investimento em empresas brasileiras não financeiras de capital aberto. Revista de Contabilidade e Organizações, 18, 26-36. https://doi.org/10.11606/rco.v7i18.55429

Cunha, L., Guidini, A. \& Klann, R. (2017). A disciplina de teoria da contabilidade e a convergência às normas internacionais de contabilidade: percepção dos professores dos programas de pós-gradução stricto sensu em Contabilidade. Pensar Contábil, 19(68), 4-16. http://www.spell.org.br/documentos/ver/45603 
Da Cunha, A., Gomes, V. \& Veras, M. (2013). Adoção do valor justo para os ativos biológicos: análise de sua relevância em empresas brasileiras, Revista Universo Contábil, 9(4),110-127. http://www.spell.org.br/documentos/ver/2 0056

Dalmutt, S., Mazzioni, S., Bordignon, A. \& Guabiani, C. (2014). Tratamento contábil dos ativos biológicos e produtos agrícolas em Cooperativas de Santa Catarina, Revista de Administração, Contabilidade e Sustentabilidade, 4(3), 42-61. http://www.spell.org.br/documentos/ver/38195

Dantas, J., Rodrigues, F., Niyama, J. \& Mendes, P. (2010). Normatização contábil baseada em princípios ou em regras? Benefícios, custos, oportunidades e riscos. Revista de Contabilidade e Organizaçôes, 4(9), 3-29. https://doi.org/ 10.11606/rco.v4i9.34765

Daza, J. (2015). Análisis de la interrelación crecimiento-rentabilidad en Brasil. Tourism \& Management Studies, 11(2), 182-188. http://dx.doi.org/10.18089/tms.2015.11222

De Aguiar, C. \& Hiroshi, S. (2017). Previsão dos analistas e adoção dos padrões IFRS em petrolíferas mundiais. Revista Universo Contábil, 13(2), 6-24. http://dx.doi.org/10.4270/ruc.2017206

De Menesse, F., Rodrigues, V. \& Vasconcelos, P. (2013). Determinantes do nível de Disclosure de ativos intangíveis em empresas brasileira, BASE - Revista de Administração e Contabilidade da Unisinos, 10(2), 142-153. http://ww w.spell.org.br/documentos/ver/10609

Dias, G., Siqueira, P. \& Beuren, I. (2014). Conformidade do Disclosure Obrigatório dos ativos intangíveis e práticas ram. Revista de administração Mackenzie, 15(5), 140-170. http://dx.doi.org/10.1590/1678-69712014

Dias, W., Cunha, J., \& Mário, P. (2009). Plano de incentivo em opções de ações e a harmonização contábil: estudo do nível de disclosure das empresas brasileiras após o CPC 10. Pensar Contábil, 11(46), 29-38. http://www.spell. org.br/documentos/ver/111

Domingos, S., Lima, S. \& Ponte, V. (2013). Income Smoothing: um estudo após a adoção do I FRS no Brasil. Contextus - Revista Contemporânea de Economia e Gestäo, 11(2), 181-199. http://www.spell.org.br/documentos/ver/255 13

Einsweiller, A. \& Fischer, A. (2013). Efeitos da aplicação de valor justo no ativo biológico de uma empresa do ramo de celulose e papel. Revista Catarinense da Ciência Contábil, 12(37), 24-34. http://www.spell.org.br/documen tos/ver $/ 33225$

Estima, I. \& Mota, M. (2015). Principais Consequências da Adoção das IFRS: Análise da literatura existente e sugestões para investigação futura. Contabilidade \& Finanças, 26(68), 126-139. http://dx.doi.org/10.1590/1808-057x2 01500090

Fé Júnior, A., Nakao, S., \& Ribeiro, M. (2015). Reações do Mercado Acionário na Primeira Divulgação Financeira em IFRS do Setor Bancário Brasileiro: um estudo de evento. Contextus - Revista Contemporânea de Economia e Gestão, 13(3), 81-110. http://www.spell.org.br/documentos/ver/39226

Fé, R., Vaz de Lima, D., \& Oliveira, L. (2012). Processo de reconhecimento e mensuração do ativo imobilizado no setor público face aos padrões contábeis internacionais: um estudo de caso na Anate. Revista Universo Contábil, 8(3), 62-81. http://www.spell.org.br/documentos/ver/7844

Fernandes, L., Santos, F., Pereira, R., \& Szuster, N. (2008). Uma abordagem sobre a harmonização entre a contabilidade brasileira e a internacional: o caso da parada programada. Revista Contemporânea de Contabilidade, 5(9), 49-70. http://www.spell.org.br/documentos/ver/35384

Figlioli, B., Lemes, S., \& Lima, F. (2017). IFRS, Sincronicidade e crise financeira: a dinâmica da informação contábil para o mercado de capitais brasileiro. Revista Contabilidade \& Finanças - USP, 28(75), 326-343. http://dx.doi .org/10.1590/1808-057x201704450

Francis, J., Lafond, R., Olsson, P., \& Schipper, K. (2005). The market pricing of accruals quality. Journal of Accounting and Economics, 39(2), 295-327. https://doi.org/10.1016/j.jacceco.2004.06.003

Fuji, A. (2008). Contabilização de Títulos e Valores Mobiliários: uma comparação entre as normas brasileiras, do FASB e do IASB. Revista Contabilidade \& Finanças - USP, 19(47), 112-123. http://www.spell.org.br/documentos/ ver/6351 
Gómez, M. (2013). El reconocimiento contextual y el aprendizaje de las publicaciones internacionales en contabilidad. Cuadernos de Contabilidad, 14(34), 273-284. http://revistas.javeriana.edu.co/index.php/cuacont/article/view 16078.

Gonçalves, K., Conegliam, L., \& Carmo, C. (2017). Value relevance das propriedades para investimento: evidências do mercado de capitais brasileiro. Contabilidade, Gestão e Governança, 20(1), 2-19. http://dx.doi.org/10.2171 4/1984-3925_2017v20n1a1

Grecco, M. (2013). O efeito da convergência brasileira às IFRS no gerenciamento de resultados das empresas abertas brasileiras não financeiras. Brazilian Business Review, 10(4), 117-140. http://www.spell.org.br/documentos/ve r/ 17676

Haveroth, J., Nez, E. Bilk, \& Klann, R. (2017). Características e perspectivas internacionais dos diversos grupos de interesses em relação à IFRS SME's. Revista de Educação e Pesquisa em Contabilidade, 11(4), 430-450. http://d x.doi.org/10.17524/repec.v11i4.1636

Homero, P. F. (2017). A Constituição do campo científico e a baixa diversidade da pesquisa contábil brasileira. REPEC, Brasilia, 11(3), 314-328. http://dx.doi.org/10.17524/repec.v11i3.1565

Ito, E., Mendes, P., \& Niyama, J. (2008). Controle de qualidade dos serviços de auditoria independente: um estudo comparativo entre as normas brasileiras e as normas internacionais. Contabilidade, Gestão e Governança, 11(1-2), 312-328. http://www.spell.org.br/documentos/ver/8449

Joia, R. \& Nakao, S. (2014). Adoção de IFRS e gerenciamento de resultado nas empresas brasileiras de capital aberto. Revista de Educação e Pesquisa em Contabilidade, 8(1), 22-38. http://www.spell.org.br/documentos/ver/28993

Klann, R. \& Beuren, I. (2010). Reflexos das divergências entre IFRS e US GAAP na evidenciação contábil. Advances in Scientific and Applied Accounting, 3(1), 2-40. http://www.spell.org.br/documentos/ver/14488

Klann, R., Popik, F., Kreuzberg, F. \& Gonçalves, N. (2014). Fatores relacionados ao nível de divulgação de ativos intangíveis após a adoção das IFRS por Empresas do IGC da BM\&FBOVESPA. Revista Catarinense da Ciência Contábil - CRCSC, 13(38), 37-51. http://www.spell.org.br/documentos/ver/33220

Klein, L. \& Almeida, L. (2017). A influência dos fatores contingenciais na adoção de práticas de contabilidade gerencial nas indústrias Paranaenses. Revista Universo Contábil, 13(3), 90-119. http://dx.doi.org/10.4270/ruc.2017320

Lafond, R. \& Watts, L. (2008). The information role of conservatism. The Accounting Review, 83(2), 447-478. http:/ /dx.doi.org/10.2308/accr.2008.83.2.447

Lara, G., Osma, G., \& Penalva, F. (2014). Information consequences of accounting conservatism. European Accounting Review, 23(2), 173-198. https://doi.org/10.1080/09638180.2014.882263

Lima, L. (2011). A representação das múltiplas dimensões paradigmáticas no estudo da administração: um ensaio sobre os limites contidos nas defesas paradigmáticas excludentes. Revista de Administração Contemporânea, 15(2), 198-208. https://doi.org/10.1590/S1415-65552011000200003.

Lopes, A. \& Alencar, R. (2010). Disclosure and cost of equity capital in emerging markets: the Brazilian case. International Journal of Accounting, 45(4), 443-464. https://doi.org/10.1016/j.intacc.2010.09.003

Loureiro, D., Gallon, A., \& Luca, M. (2011). Subvenções e Assistências Governamentais (Sag): evidenciação e rentabilidade das maiores empresas brasileiras. Revista de Contabilidade e Organizações, 5(13), 34-54. http://w ww.spell.org.br/documentos/ver/4943

Lourenço, I. \& Branco, M. (2015). A Governança Corporativa e o efeito da adoção das IFRS: o caso brasileiro. Revista Universo Contábil, 11(1), 157-172. http://dx.doi.org/10.4270/ruc.2015108

Luzivalda, G., \& Edilson, P. (2017). Meta-análise dos Efeitos da Adoção das IFRS na Qualidade da Informação Contábil no Brasil. Revista de Contabilidade do Mestrado em Ciências Contábeis da UERJ, 22(1), 28-48. http:/ /www.spell.org.br/documentos/ver/48337

Macedo, M., Machado, M., \& Machado, M. (2013). Análise da relevância da informação contábil no Brasil num contexto de convergência às normas internacionais de contabilidade. Revista Universo Contábil, 9(1), 65-85. ht tp://www.spell.org.br/documentos/ver/9664

Machado, M. \& Nakao, S. (2014). Influência das diferenças culturais, econômicas e sociais na adoção das IFRS. Revista Universo Contábil, 10(1), 104-125. http://www.spell.org.br/documentos/ver/29020 
Macías, H. \& Cortés, J. (2009). El campo de la investigación contable: oportunidades para los investigadores colombianos. Cuadernos de Contabilidad, 10(26), 21-50. http://revistas.javeriana.edu.co/index.php/cuacont/a rticle/view/3216

Magalhães, R., Pinheiro, L., \& Lamounier, W. (2011). Fatores que favorecem a compreensão da extensão da divulgação sobre partes relacionadas - estudo nas companhias listadas no novo mercado da BM\&FBovespa. Sociedade, Contabilidade e Gestão, 6(3), 22-37. http://www.spell.org.br/documentos/ver/33869

Maia, H., Formigoni, H., \& Silva, A. (2012). Empresas de auditoria e o compliance com o nível de evidenciação obrigatório durante o processo de convergência às Normas Internacionais de Contabilidade no Brasil. Revista Brasileira de Gestão de Negócios, 14(44), 335-352. http://www.spell.org.br/documentos/ver/8694

Major, M. (2017). O positivismo e a pesquisa 'alternativa' em Contabilidade. Revista Contabilidade \& Finanças, 28(74), 173-178. http://dx.doi.org/10.1590/1808-057x201790190.

Mantovani, E. \& Almeida, F. (2014). A Contabilização do Ativo Intangível nas 522 Empresas Listadas na BM\&FBOVESPA. Revista de Administ ração e Inovação, 11(3), 311-328. http://www.spell.org.br/documento s/ver/34305.

Margarete, L. \& Klann, R. (2015). Disclosure obrigatório de ativos intangíveis das companhias listadas nos níveis de governança corporativa da Bm\&FBovespa. Pensar Contábil, 62(45), 45-54. http://www.spell.org.br/documen tos/ver/37280

Mejía, E., Mora, G., \& Montes, A. (2013). Adscripción de la contabilidad en la estructura general del conocimiento. Cuadernos de Contabilidad, 14(34), 159-187. http://revistas.javeriana.edu.co/index.php/cuacont/article/view 16072

Mendonça, O., Riccio, E., \& Sakata, M. (2009). Dez anos de pesquisa contábil no Brasil: análise dos trabalhos apresentados nos ENANPADs de 1996 a 2005. Revista de Administração de Empresas, 49(1), 62-73. https://d oi.org/10.1590/S0034-75902009000100008.

Moraes, F. \& Carvalho, K. (2014). Escolhas contábeis na adoção inicial das Normas Internacionais de Contabilidade no Brasil: direcionadores da aplicação do custo atribuído para ativos imobilizados. Revista Contabilidade Vista \& Revista, 25(3), 38-56. http://www.spell.org.br/documentos/ver/35254

Moreira, C. \& Lima, A. (2003). A evidenciação dos derivativos no Brasil: uma tentativa de convergência para procedimentos internacionais. Revista de Contabilidade do Mestrado em Ciências Contábeis da UERJ, 8(1), 81-97. http://www.spell.org.br/documentos/ver/27234

Moura, A. \& Coelho, A. (2016). Impacto de mudanças em padrões contábeis em indicadores de endividamento de firmas: evidências no Brasil. Brazilian Business Review, 13(5), 27-51. http://dx.doi.org/10.15728/bbr.2016.13 .5 .2

Murcia, F., Silva, A., Barreto, E., \& Carvalho, L. (2008). Conjectures regarding the adoption of fair value measurements in Brazil. Revista Contemporânea de Contabilidade, 5(9), 11-28. http://www.spell.org.br/documentos/ver/353 82

Nascimento, A., Junqueira, E., \& Martins, G. (2010). Pesquisa acadêmica em contabilidade gerencial no Brasil: análise e reflexões sobre teorias, metodologias e paradigmas. Revista de Administração Contemporânea, 14(6), 1113-1133. https://doi.org/10.1590/S1415-65552010000700008

Niyama, J., Barbosa, E., Cavalcanti, R. \& Leite, D.(2002). Contabilização das operações de leasing: avaliação do nível de aderência da Norma Técnica 10.2 do Conselho Federal de Contabilidade à luz das Normas Internacionais de Contabilidade do IASB. Contabilidade Vista \& Revista, 13(3), 21-34. http://www.spell.org.br/documentos/v $\mathrm{er} / 25175$

Nunes, I., Funchal, B., \& Beiruth, A. (2017). A Lei de Falências e a Estratégia de endividamento das empresas brasileiras: um estudo sobre o uso da Teoria do Pecking Order e da Teoria do trade-off. Sociedade, Contabilidade e Gestão, 12(1), 26-43. http://www.spell.org.br/documentos/ver/45035

Oliveira, N., Nakao, S., \& Nardi, P. (2017). Análise da Influência das firmas de auditoria na divulgação de informações em notas explicativas. BASE - Revista de Administração e Contabilidade da UNISINOS, 14(2), 139-154. http: //dx.doi.org/10.4013/base.2017.142.05 
Perea, S. (2015). Perspectiva crítica del valor razonable en el marco de la crisis financiera. Cuadernos de Contabilidad, 16(42), 761-779. http://revistas.javeriana.edu.co/index.php/cuacont/article/view/17392

Pereira, R. \& Marques, J. (2009). Comentários sobre a evidenciação das operações de arrendamento mercantil no contexto da convergência com as práticas contábeis internacionais: o caso da Petróleo Brasileiro S/A. Pensar Contábil, 11(43), 45-54. http://www.spell.org.br/documentos/ver/100

Peruchena, J., Shneider, L., \& Kronbauer, L. (2015). Estudo das normas e práticas contábeis adotados pelas empresas industriais do Mercosul e Comunidade Andina: convergência referente a mensuração e evidenciação das propriedades para investimento. Revista de Contabilidade do Mestrado em Ciências Contábeis da UERJ, 19(3), 2-18. http://www.spell.org.br/documentos/ver/37262

Pires, C. \& Decourt, R. (2015). Os impactos da fase final de transição para o IFRS no Brasil. Revista Brasileira de Gestão de Negócios, 17(54), 736-750. http://dx.doi.org/10.7819/rbgn.v17i54.1475

Pires, J., Pereira, M., Katsumi, J. \& Tiburcio, C. (2017). Adoção ao padrão IFRS e earnings quality: a persistência do lucro das empresas listadas na BM\&FBovespa. Revista de Contabilidade e Organizações, 29, 46-55. http://dx.d oi.org/10.11606/rco.v11i29.125846.

Pito, T. \& Barros, J. (2016). A aplicação das normas contabilísticas de "justo valor” nas demonstrações de resultados das empresas integradas no Portuguese Stock Index-20 (PSI-20). Revista Brasileira de Gestão de Negócios, 18(59), 67-86. http://www.scielo.br/scielo.php?pid=S1806-48922016000100067\&script

Poli, A., Doná, L. \& Neumann, M. (2014). Disclosure da perda por impairment aplicado ao ativo imobilizado: análise nas companhias do IBrX-501. Ref Cont, 33(1), 1-18. http://dx.doi.org/10.4025/enfoque.v33i1.21557

Ramos, D. \& Da Silva P. (2017). Nível de Disclosure do CPC 29 Ativos Biológicos: Análise dos fatores determinantes nas companhias brasileiras. Contabilidade, Gestão e Governança, 20(1), 38-54. http://dx.doi.org/10.21714/19 84-3925_2017v20n1a3

Rech, I., Vieira, I., Ribeiro, \& Oliveira, J. (2008). Impostos diferidos na atividade pecuária originados da avaliação dos ativos biológicos pelo valor justo: um estudo de seu reconhecimento e evidenciação nas maiores propriedades rurais do Estado de Mato Grosso. Revista Universo Contábil, 4(2), 42-58. http://www.spell.org.br/documento s/ver/6155

Rezende, C., Almeida, N., \& Lemes, S. (2015). Impacto das IFRS na assimetria de informação evidenciada no mercado de capitais brasileiro. Revista de Contabilidade e Organizaçôes, 9(24), 18-30. http://dx.doi.org/10.11606/rco.v 9i24.55524

Rosa, A. \& Alcadipani, R. (2013). A terceira margem do rio dos estudos críticos sobre administração e organizações no Brasil: repensando a crítica a partir do pós-colonialismo. Revista de Administração Mackenzie, 14(6), 185-215. h ttps://doi.org/10.1590/S1678-69712013000600009

Rueda, G. (2010). El papel de la contabilidad ante la actual realidad económica, social y política del país. Más allá de la convergencia de prácticas mundiales. Cuadernos de Contabilidad, 11(28), 149-169. http://revistas.javeriana. edu.co/index.php/cuacont/article/view/3202

Ruiz, G. (2008). Las normas internacionales de contabilidad y la contabilidad creativa. Cuaderno de Contabilidad, 9(24), 251-301. http://revistas.javeriana.edu.co/index.php/cuacont/article/view/3250

Sá, F. \& Malaquias, R. (2012). Análise da percepção dos discentes do curso de graduação em ciências contábeis sobre expressões de incerteza contidas nos pronunciamentos emitidos pelo comitê de pronunciamentos contábeis. Revista de Contabilidade e Organizaçôes, 6(14), 77-105. http://www.spell.org.br/documentos/ver/7630

Salazar, E. (2009). Comparación entre la Norma Internacional de Información Financiera para pequeñas y medianas entidades (NIIF para PYME) y la normatividad colombiana. Cuadernos de Contabilidad, 10(27), 361-430. htt p://revistas.javeriana.edu.co/index.php/cuacont/article/view/3213

Sánchez, A. \& Salazar, E. (2010). Convergencia contable internacional: hacia un nuevo modelo de contabilización de arrendamientos. Cuaderno de Contabilidad, 11(28), 67-121. http://revistas.javeriana.edu.co/index.php/cuacon t/article/view/3177 
Santiago, J., Cavalcante, P., \& Paulo, E. (2015). Análise da persistência e conservadurismo no processo de convergência internacional nas empresas de capital aberto do setor de construção no Brasil. Revista Universo Contábil, 11(2), 174-195. http://dx.doi.org/10.4270/ruc.2015217

Santos, E. \& Cia, J. (2009). Impactos esperados da harmonização internacional no lucro das empresas brasileiras, na proxy dos ajustes BRGAAP/USGAAP reportados pelas emissoras de ADRS na NYSE. Revista de Contabilidade e Organizaçôes, 3(6), 57-80. http://www.spell.org.br/documentos/ver/4881

Santos, E. (2012). Análise dos impactos dos CPCS da primeira fase de transição para o IFRS no Brasil: um exame dos ajustes aos resultados nas DFPS de 2008. Revista de Contabilidade e Organizaçôes, 6(15), 23-43. http://www.s pell.org.br/documentos/ver/8540

Santos, E. (2015). Impacto total da implementação do IFRS no lucro e patrimônio líquido das empresas brasileiras. BASE - Revista de Administração e Contabilidade da UNISINOS, 12(3), 225-241. http://dx.doi.org/10.4013/ base.2015.123.05

Santos, L., Lima, G., Freitas, S., \& Lima, I. (2011). Efeito da Lei 11.638/07 sobre o conservadurismo condicional das empresas listadas BM\&FBovespa. Revista Contabilidade \& Finanças - USP, 22(56), 174-188. http://dx.doi.or g/110.1590/S1519-70772011000200004.

Santos, O. \& Silva, P. (2014). Os métodos contábeis dos esforços bem-sucedidos capitalização total: um estudo sob a perspectiva do Value Relevance. Enfoque Reflexão Contábil, 33(2), 121-138. http://dx.doi.org/10.4025/enfo que.v33i2.22307

Sayed, S., Souza, B., Costa, J. \& Tancini, G. (2013). Simulação dos impactos da alteração da norma internacional de instrumentos financeiros (IFRS 9) nos maiores bancos brasileiros. Sociedade, Contabilidade e Gestão, 8(1), 50-63. http://www.spell.org.br/documentos/ver/33736

Scalzer, R., Beiruth, A., \& Reina, D. (2017). Empresas Estatais e Conservadurismo Contábil: Uma Análise das Empresas da BM\&FBovespa. REAd. Revista Eletrônica de Administração, 23(2), 333-350. http://dx.doi.org/1 $0.1590 / 1413.2311 .076 .62934$

Schrickel, W. (1997). Demonstraçôes financeiras. Abrindo a caixa-preta: como interpretar balanços para a concessão de empréstimos. São Paulo: Editorial Atlas.

Schwarz, D., Barbosa, A., \& Freitas, M. (2019). Fatores determinantes da divulgação voluntária 'Web-Based' das companhias brasileiras de saneamento básico. Revista Evidenciação Contábil \& Finanças, 7(2), 118-141. https:/ /doi.org/10.22478/ufpb.2318-1001.2019v7n2.39791

Silva Filho, G., Lucena, W. \& Leite Filho, P. (2017). Efeito do reconhecimento do Fair Value Accounting nas decisões de venda diante da influência dos vieses cognitivos: um estudo à luz da Prospect Theory. PensarContábil, 19(69), 40-53. http://www.spell.org.br/documentos/ver/46942

Silva, A., Heinzen, C., Klann, R. C., \& Lemes, S. (2018). Relação entre o Conservadurismo Contábil e a Relevância das Informações. Advances in Scientific and Applied Accounting, 11(3), 502-516. http://dx.doi.org/10.14392/a saa.2018110308

Silva, A., Macedo, M. \& Marques, J. (2012). Análise da relevância da informação contábil no setor brasileiro de energia elétrica no período de 2005 a 2007: uma discussão com foco nas variáveis II, FCO e EBITDA. Revista Universo Contábil, 8(2), 6-24. http://dx.doi.org/10.4270/ruc.2012210

Silva, C., Ramos, F., Mendonça, K., \& Nascimento, S. (2003). Uma abordagem sobre as transformações de sociedades e a necessidade de harmonização de normas contábeis. Contabilidade Vista \& Revista, 14(ed. especial), 123-143. http://www.spell.org.br/documentos/ver/25241

Silva, F., Couto, G., \& Cordeiro, R. (2009). Measuring the impact of International Financial Reporting Standards (IFRS) to financial information of Portuguese companies. Revista Universo Contábil, 5(1), 129-144. http://dx .doi.org/10.4270/ruc.20095

Silva, J. (2011). Demonstrações contábeis consolidadas: uma análise comparativa das Normas Brasileiras (BRGAAP) e Internacionais (IFRS). Pensar Contábil, 13(51), 26-34. http://www.atena.org.br/revista/pensarcontabil/artic le/view/962 
Silva, J., Bonfim, M., Niyama, J. \& Silva, C. (2017). Adoção ao Padrão IFRS e Earnings Quality: a persistência do lucro das empresas listadas na BM\&FBovespa. Revista de Contabilidade e Organizaçôes, 11(29), 46-55. http://dx.doi .org/10.11606/rco.v11i29.125846

Silva, J., Borges, T., Gonçalves, R. \& Nascimento, D. (2017). Convergência ao Padrão IFRS e suavização de resultados em Empresas de Energia Elétrica. BASE - Revista de Administração e Contabilidade da UNISINOS, 14(4), 281-296. http://dx.doi.org/10.4013/base.2017.144.04

Silva, J., Fonseca, L., \& Nogueira, D. (2014). Nível de conformidade do pronunciamento CPC 28: uma análise nas companhias abertas brasileiras. Revista Catarinense da Ciência Contábil - CRCSC, 13(40), 54-66. http://dx.d oi.org/10.16930/2237-7662/rccc.v13n40p54-66

Silva, T. \& Nakao, S. (2011). Divulgação na adoção pela primeira vez de IFRS por empresas europeias de setores e sistemas jurídicos diferentes. Contabilidade Vista \& Revista, 22(2), 93-124. http://www.spell.org.br/documen tos/ver/8170

Silva, B. (2014). La información financiera como factor clave en el mercado de capitales: una reflexión sobre las modificaciones en el marco conceptual del IASB. Estrategias, 12(22), 51-62. https://www.researchgate.net/pu blication/292991727

Silveira, A., Leal, R., Barros, L. \& Carvalhal-da-Silva, A. (2009). Evolution and determinants of firm-level corporate governance quality in Brazil. Revista de Administração, 44(3), 173-189. http://www.spell.org.br/documentos/ ver/7292

Sosa, J. (2013). Reflexiones en torno a las experiencias del proceso de convergencia hacia normas internacionales de aseguramiento de la información. Cuaderno de Contabilidad, 14(36), 1079-1095. http://revistas.javeriana.edu. co/index.php/cuacont/article/view/7913

Sousa, L., Castro, L., Viana, D., Domingos, S., \& Ponte, V. (2018). Conservadurismo condicional e adoção das IFRS: Uma análise nas empresas brasileiras de capital aberto. Enfoque Reflexão Contábil, 37(3), 21-35. http://dx.doi. org/10.4025/enfoque.v37i3.35390

Souza, A. \& De Lima, D. (2014). Reconhecimento contábil da degeneração de ativos biológicos para a produção no cultivo de árvores frutíferas. Revista Contemporânea de Contabilidade, 11(22), 73-94. http://www.spell.org.br/ documentos/ver/35521

Souza, F. \& Lemes, S. (2016). Comparability of accounting choices in subsequent measurement of fixed assets, intangible assets, and investment property in South American Companies. Revista Contabilidade \& Finanças USP, 27(71), 169-184. http://dx.doi.org/10.1590/1808-057x201501480

Strouhal, J. (2009). Aplicação das Normas Contabilísticas Internacionais IAS 32, IFRS 7 e IAS 39 em prática das entidades empresariais da República Checa. Revista Universo Contábil, 5(4), 152-161. http://www.spell.org.br /documentos/ver/6221

Talaska, A. \& De Lima, D. (2016). Nível de Disclosure de ativos biológicos nas empresas listadas na BM\&Fbovespa: Análise pós-adoção do Valor Justo. Revista de Contabilidade do Mestrado em Ciências Contábeis da UERJ, 21(3), 22-39. http://www.spell.org.br/documentos/ver/44485

Tavares, M., Anjos, L. \& Paulo, E. (2014). Contribuições enviadas ao IASB/FASB referentes à revisão do draft de reconhecimento de receitas. Contextus - Revista Contemporânea de Economia e Gestão, 12(3), 35-63. http://ww w.spell.org.br/documentos/ver/32895

Torres, F. \& Rodríguez, B. (2008). La maximización de beneficios bajo los estándares internacionales de contabilidad financiera: una aproximación a las aplicaciones en las pyme. Cuaderno de Contabilidad, 4(24), 73-130. http://r evistas.javeriana.edu.co/index.php/cuacont/article/view/3244

Vale, J. \& Nakao, S. (2017). Conservadurismo incondicional nas companhias abertas brasileiras e o contexto da neutralidade tributária. Revista Contabilidade \& Finanças - USP, 28(74), 197-212. http://dx.doi.org/10.1590 /1808-057x201702450

Zonatto, V., Santos, A., Rodrigues Junior, M., \& Bezerra, F. (2011). Fatores determinantes para a adoção de padrões internacionais de contabilidade no Brasil: uma investigação em empresas públicas e privadas do Setor de Energia Elétrica. Revista de Contabilidade e Organizações, 5(12), 26-47. http://www.spell.org.br/documentos/ver/4933 
Zortea, C., Galdi, F., Monte-mor, D., \& Beiruth, A. (2017). Eficiência do mercado de capitais após a adoção da IFRS no Brasil: Aplicando o Teste de Mishkin. Revista Contemporânea de Contabilidade, 14(32), 141-156. http://dx .doi.org/10.5007/2175-8069.2017v14n32p141

\section{Notas}

* Artículo de investigación científica.

Artículo producto de la investigación amparada por el proyecto "Análise das demonstrações contábeis como subsídio ao processo decisório", código: 0036/2017 de la entidad Universidade Federal de Uberlândia. Reconocemos el apoyo de CAPES y CNPq para el desarrollo de la investigación.

\section{Licencia Creative Commons CC BY 4.0}

Para citar este artículo: Vazquez C, X., \& Miranda, G. J. (2020). Tendencias en los estudios brasileños sobre el cambio de normativa contable. Cuadernos de Contabilidad, 21. https://doi.org/10.11144/Javeriana.cc21 tebc 